Revue d'histoire de l'Amérique française

REVUE D.HISTOIRE DE L'AMÉRIQUE FRANÇAISE

\title{
La naissance au Saguenay et dans Charlevoix (1900-1950) : continuités et ruptures culturelles
}

\section{Josée Gauthier}

Volume 48, numéro 3, hiver 1995

URI : https://id.erudit.org/iderudit/305349ar

DOI : https://doi.org/10.7202/305349ar

Aller au sommaire du numéro

Éditeur(s)

Institut d'histoire de l'Amérique française

ISSN

0035-2357 (imprimé)

1492-1383 (numérique)

Découvrir la revue

Citer cet article

Gauthier, J. (1995). La naissance au Saguenay et dans Charlevoix (1900-1950) : continuités et ruptures culturelles. Revue d'histoire de l'Amérique française, 48(3), 351-373. https://doi.org/10.7202/305349ar
Résumé de l'article

Cette analyse repose sur la problématique culturelle des transferts migratoires vers de nouveaux espaces de peuplement. Le rituel associé à la naissance d'un enfant sert de fil conducteur à cette étude sur la dynamique du changement culturel. Nous nous proposons de déterminer si les mouvements migratoires à partir de la région de Charlevoix vers le Saguenay depuis la seconde moitié du XIX ${ }^{\mathrm{e}}$ siècle ont affecté certains contenus ou modèles coutumiers, et dans quelle mesure ils les influencent. Le cas échéant, on suppose que les codes culturels de Charlevoix ont pu être modifiés selon deux processus, à savoir : l'érosion (ou la contraction) et le renouvellement des rites. En plus d'éclairer certains aspects du rituel au Saguenay et dans Charlevoix, notre étude vise aussi à rechercher des éléments de différenciation spatiale dans le Nord-Est québécois, voire l'émergence possible de deux régions culturelles.
Tous droits réservés @ Institut d'histoire de l'Amérique française, 1995
Ce document est protégé par la loi sur le droit d'auteur. L'utilisation des services d'Érudit (y compris la reproduction) est assujettie à sa politique d'utilisation que vous pouvez consulter en ligne.

https://apropos.erudit.org/fr/usagers/politique-dutilisation/ 


\title{
LA NAISSANCE AU SAGUENAY ET DANS CHARLEVOIX (1900-1950) CONTINUITÉS ET RUPTURES CULTURELLES ${ }^{1}$
}

\author{
JOSÉE GAUTHIER \\ IREP \\ Université du Québec à Chicoutimi
}

\section{RÉSUMÉ}

Cette analyse repose sur la problématique culturelle des transferts migratoires vers de nouveaux espaces de peuplement. Le rituel associé à la naissance d'un enfant sert de fil conducteur à cette étude sur la dynamique du changement culturel. Nous nous proposons de déterminer si les mouvements migratoires à partir de la région de Charlevoix vers le Saguenay depuis la seconde moitié du XIX ${ }^{e}$ siècle ont affecté certains contenus ou modèles coutumiers, et dans quelle mesure ils les influencent. Le cas échéant, on suppose que les codes culturels de Charlevoix ont pu être modifiés selon deux processus, à savoir: l'érosion (ou la contraction) et le renouvellement des rites. En plus d'éclairer certains aspects du rituel au Saguenay et dans Charlevoix, notre étude vise aussi à rechercher des éléments de différenciation spatiale dans le Nord-Est québécois, voire l'émergence possible de deux régions culturelles.

\begin{abstract}
This paper is based upon the cultural problematic of migratory transfers to newly settled territories. Our purpose is to determine if, since the mid-nineteenth century, the migrations between the Charlevoix and Saguenay regions somehow altered cultural patterns and behavior. The changes affecting the birth ritual are the main subject of this study of cultural dynamics. The study suggests that cultural codes of the Charlevoix might have been modified by two processes: erosion (or contraction) and renewal. In addition to analysing rituals as such, the paper attempts to capture indications of spatial stratification and, possibly, the emergence of two distinct cultural regions in the North-East of the province of Quebec.
\end{abstract}

1. Ce texte présente les principaux résultats d'un mémoire de maîtrise déposé à l'Université du Québec à Chicoutimi. L'étude, qui s'inscrivait dans un projet de l'Institut interuniversitaire de recherches sur les populations (IREP), a bénéficié d'une bourse de la Fondation de l'Université du Québec. J'aimerais remercier le directeur de l'Institut, monsieur Gérard Bouchard ainsi qu'Anne-Marie Desdouits, Françoise Loux et Diane Gervais pour leurs conseils et commentaires.

RHAF, vol. 48, $\mathrm{n}^{\circ}$ 3, hiver 1995 


\section{I - CADRE HISTORIQUE ET CONCEPTUEL}

Le contexte de cette enquête est celui d'une région de colonisation ouverte au peuplement à compter de 1838. Durant les premières décennies, un important corridor migratoire unissait les régions du Saguenay et de Charlevoix. D'ailleurs, entre 1838 et 1900 , une partie importante des surplus démographiques de Charlevoix alimenta les migrations en direction du Saguenay ${ }^{2}$. L'importance de ce courant migratoire entre les deux régions les désigne pour une étude sur les effets des transferts de population sur la dynamique culturelle ${ }^{3}$. L'intérêt d'une telle étude est d'en évaluer les conséquences, en essayant de voir si les conditions inhérentes à la colonisation n'auraient pas entraîné une rupture ou une différenciation dans les modèles coutumiers. Plus particulièrement, on se demande si le rituel charlevoisien a été reproduit intégralement au Saguenay ou s'il a subi des modifications causées par les traumatismes associés à de tels transferts. Pour cerner la dynamique de changement qui a pu en découler, nous reconstituerons les séquences du rituel (grossesse, accouchement, relevailles, etc.) entourant la naissance physique et sociale d'un enfant au Saguenay. De cette manière, il sera possible de comparer les contenus coutumiers de ces deux régions afin de tester l'hypothèse selon laquelle les déplacements de population seraient à l'origine de bouleversements au sein de ce rituel. Selon cette hypothèse, les modifications apportées au code coutumier auraient été effectuées par le biais de deux vecteurs de changements, l'un étant susceptible d'en provoquer la «déritualisation», l'autre pouvant, au contraire, contribuer à le renouveler, le diversifier. En effet, la première forme de mutation ayant pu affecter les contenus coutumiers relatifs à la nais-

2. Entre 1852 et 1869 , plus de $80 \%$ des immigrants provenaient de cette région. Pour une esquisse de l'histoire du peuplement, se reporter à Gérard Bouchard, «Le peuplement blanc», Christian Pouyez, Yolande Lavoie, et al., Les Saguenayens. Introduction à l'histoire des populations du Saguenay, XVI ${ }^{e}-X X^{e}$ siècles (Québec, Presses de l'Université du Québec, 1983), 138; Raymond Roy, Gérard Bouchard, Manon Declos, «La première génération de Saguenayens: provenance, apparentement, enracinement», Cahiers québécois de démographie, 17,1 (printemps 1988): 116; Danielle Gauvreau, Mario Bourque, «Mouvements migratoires et familles: le peuplement du Saguenay avant 1911", Revue d'histoire de l'Amérique française, 42,2 (automne 1988): 167-192.

3. Pour un exposé détaillé sur ce sujet, on lira Gérard Bouchard, «Sur les dynamiques culturelles des régions de peuplement», Canadian Historical Review, LVXIII,4 (1986): 473-490; Gérard Bouchard, Josée Gauthier, Marie-Josée Huot, «Permanences et mutations dans l'histoire de la culture paysanne québécoise», Gérard Bouchard, dir., avec la collaboration de Serge Courville, La construction d'une culture. Le Québec et l'Amérique française (Sainte-Foy, Presses de l'Université Laval, 1993), 261-305. Aussi: Josée Gauthier, Évolution des pratiques coutumières entourant la naissance au Saguenay et dans Charlevoix (1900-1950), mémoire de maîtrise (études régionales), Université du Québec à Chicoutimi, 1991, 288 pages. 
sance est la déperdition. Par ce terme, on entend la mise en place d'un processus d'érosion ou de contraction entraînant l'émergence d'un rituel beaucoup plus dépouillé que par le passé. Ce dernier se révélerait alors dépourvu d'un certain nombre d'éléments (conduites, normes) servant à programmer et à prescrire les gestes à poser, les comportements à adopter. L'absence de rites, pratiques et croyances fait que, dans certaines circonstances, les individus doivent improviser «autour d'un canevas très rudimentaire et stéréotypé ${ }^{4}$. En somme, le rituel saguenayen aurait alors comporté moins d'éléments codés et symboliques que celui de Charlevoix. En contrepartie, la deuxième forme de changement, soit le renouvellement, a pu favoriser l'expansion et le réaménagement de certains traits coutumiers. Ce processus aurait contribué à la diversification du rituel, en permettant l'ajout (par innovation et emprunt) de rites, la redéfinition (dans les finalités et fonctions du rite) et/ou le remplacement de certains autres. Sur le plan de la comparaison Saguenay/Charlevoix, un autre apport visé par cette étude était d'amener certains éléments de réponse à l'hypothèse de Gérard Bouchard sur la stratification de l'espace culturel québécois. Sous le rapport des conduites et des représentations symboliques, cette piste de recherche remet en question l'existence d'aires culturelles calquées sur les régions administratives. Il faudrait plutôt songer à juxtaposer au découpage géographique des régions des unités culturelles les regroupant selon un axe nord-sud et est-ouest ${ }^{5}$.

Pour mettre à l'épreuve ces hypothèses, nous avons procédé par entrevues à l'aide d'un questionnaire inspiré de celui élaboré par JeanPhilippe Gagnon ${ }^{6}$. Cet instrument de travail contient huit parties: $1^{\circ}$ la sexualité; $2^{\circ}$ la grossesse; $3^{\circ}$ l'accouchement; $4^{\circ}$ les relevailles; $5^{\circ}$ l'allaitement; $6^{\circ}$ le nouveau-né; $7^{\circ}$ le baptême; $8^{\circ}$ l'enfant suivant. À l'aide du questionnaire, 20 entrevues ont été réalisées au Saguenay auprès de femmes du troisième âge. Les personnes susceptibles de répondre au questionnaire furent choisies en fonction des cinq critères suivants: tous les informateurs devaient être de sexe féminin; $1^{\circ}$ ces femmes avaient eu des enfants ou, à tout le moins, avaient été en rapport immédiat avec la naissance d'un enfant, par exemple à titre de sage-femme ou d'assistante du médecin; $2^{\circ}$ leur date de naissance était

4. Gérard Bouchard et al., op. cit., 266.

5. Ibid., 298; Gérard Bouchard, «La région culturelle: un concept, trois objets. Essai de mise au point (commentaire)», Fernand Harvey, dir., La région culturelle. Problématique interdisciplinaire (Sainte-Foy, Presses de l'Université Laval, 1994), 111-122.

6. Jean-Philippe Gagnon, Rites et croyances de la naissance à Charlevoix (Québec, Leméac, 1979), 137-150; Josée Gauthier, Gérard Bouchard, Les rites de la naissance au Saguenay et dans Charlevoix: questionnaire d'entrevue (IREP, 1987), 33 p. (Document II-C-125). 
antérieure à $1915 ; 3^{\circ}$ leur mariage et la naissance de leur dernier enfant avaient eu lieu avant $1950 ; 5^{\circ}$ il fallait qu'elles soient nées au Saguenay; $5^{\circ}$ leurs ancêtres devaient venir de Charlevoix, au moins du côté maternel ${ }^{7}$.

Par ailleurs, les données saguenayennes ont été comparées avec celles de Gagnon. Toutefois, des 26 entrevues effectuées par ce dernier, seulement neuf ont pu être retenues dans le cadre de l'enquête, le sexe et l'âge des 16 autres témoins les ayant éliminés. Au total, le présent article fait donc état des résultats obtenus par le biais de 29 entretiens, soit 19 pour le Saguenay et 10 pour Charlevoix ${ }^{8}$.

Ces entretiens ont permis de reconstituer le rituel de la naissance, pour lequel d'ailleurs nous reprenons ici la définition de Gérard Bouchard qui désigne par ce concept:

un ensemble de normes et de coutumes (...) se rapportant à une situation, à une période ou à un événement donné et qui font que le déroulement de l'action, au lieu d'être livré au hasard et à l'improvisation, fait l'objet d'une procédure plus ou moins poussée et plus ou moins contraignante, prescrite par la culture. Ce déroulement est donc soumis à une série de règles et de modèles qui prévoient à la fois les dispositions à prendre, les actes que chacun doit poser et les modalités de leur exécution?.

Dans le cas présent, le rituel renvoie aux étapes entourant la naissance (grossesse, accouchement, allaitement, etc.). Celles-ci comportent des représentations symboliques, des gestes profanes, des rites, des croyances et des coutumes qui viendront témoigner de la dynamique de changement culturel.

\section{II - LA NAISSANCE AU SAGUENAY}

De sa conception jusqu'à son baptême, l'enfant faisait l'objet de mille et une attentions. Que ce soit par les interdits et les prescriptions formulés par l'entourage de la future mère, par les présages énoncés

7. À partir de la banque de données de l'IREP, nous avons calculé la profession dominante du père de chaque témoin. Dans 14 cas sur 19 , le père de l'informatrice était cultivateur tandis que dans les cinq autres cas, la profession du père était soit journalier (2/5), menuisier $(1 / 5)$, navigateur $(1 / 5)$ et cordonnier $(1 / 5)$.

8. Il est à noter que le questionnaire de Gagnon sur Charlevoix était moins détaillé que celui du Saguenay. Par conséquent, les entrevues du Saguenay ont pu paraître plus riches que celles de Charlevoix.

9. Gérard Bouchard, op. cit., 266. Sur cette notion, voir aussi Jean Maisonneuve, Les rituels (Paris, Presses universitaires de France, 1988), 6; Nicole Sindzingre, «Rituel», Encyclopadia Universalis (Paris, 1989), 68-71; Martine Segalen, Amours et mariages dans l'ancienne France (Paris, Berger-Levrault, 1981), 22. 
à l'endroit du nouveau-né, etc., tous ces comportements témoignent de l'importance que prenait l'enfant dans la société saguenayenne de la première moitié du $\mathrm{XX}^{\mathrm{e}}$ siècle. Tout en faisant ressortir ce phénomène, cette partie du travail décrira les comportements coutumiers relatifs à la naissance et soulignera la présence ou l'absence de modalités régissant telle et telle composante du rituel.

\section{A - Sexualité et grossesse}

Au sein de la société saguenayenne des années 1920-1940, la sexualité était un sujet tabou ${ }^{10}$. Lorsqu'arrivait le moment du mariage, les futurs époux recevaient très peu d'information sur ce sujet ${ }^{11}$. Dans le cas de nos témoins, ces renseignements faisaient surtout référence aux obligations de la femme envers son époux ${ }^{12}$. Pour le reste, ces femmes ne savaient guère ce qui allait se passer une fois mariées. Cette absence marquée de renseignements sur la question sexuelle incitait certains couples à retarder la consommation du mariage. D'ailleurs, aucune règle ou coutume particulière ne semblait associée à la consommation du mariage lors de la nuit de noces.

Du reste, la sexualité n'était pas le seul sujet sur lequel la morale de l'époque imposait le silence. Il en était de même de la grossesse; la plupart de ces femmes ignoraient la durée de la grossesse et la façon dont naissaient les enfants. Les propos d'une informatrice nous pous-

10. Le tabou entourant la question sexuelle constitue un premier exemple de l'influence qu'ont exercée les autorités religieuses au sein de la culture populaire. Cette influence s'étend d'ailleurs à d'autres aspects du rituel de la naissance. En outre, il ne s'agit pas d'une donnée récente. Plusieurs auteur(e)s ont fait état de ce phénomène en l'étendant à plus d'un domaine. Sur cet énoncé, on se reportera entre autres à Gérard Bouchard, «Les prêtres, les capitalistes et les ouvriers à Chicoutimi (1896-1930)», Mouvement social, 112 (juillet-septembre 1980): 6-23; Jacques Gélis, L'arbre et le fruit. La naissance dans l'Occident moderne (XVI'-XIXe siècles) (Paris, Fayard, 1984); Guy Laperrière, «Religion populaire, religion de clercs? Du Québec à la France, 1972-1982», Benoît Lacroix et Jean Simard, dirs., Religion populaire, religion de clercs? (Québec, Institut québécois de recherche sur la culture, 1984), 24-30; Horace Miner, SaintDenis: un village québécois (Montréal, Hurtubise HMH, 1985), 167-194; Hélène Laforce, Histoire de la sage-femme dans la région de Québec (Québec, Institut québécois de recherche sur la culture, 1985); Anne-Marie Desdouits, La vie traditionnelle au pays de Caux et au Canada français. Le cycle des saisons (Québec/Paris, Presses de l'Université Laval/Centre national de la recherche scientifique, 1987) et Le monde de l'enfance. Traditions du pays de Caux et du Québec (Québec/Paris, Presses de l'Université Laval/Centre national de la recherche scientifique, 1990); Andrée Lévesque, La norme et les déviantes. Des femmes au Québec pendant l'entre-deux-guerres (Montréal, Éditions du Remue-ménage, 1989).

11. Quatre informatrices seulement avaient reçu un peu d'information sur la sexualité avant leur mariage, soit deux de leur mère et deux du curé de la paroisse.

12. Deux informatrices ont rapporté que la femme ne devait pas se refuser à son mari Le prêtre avait également expliqué à deux autres témoins que leur corps ne leur appartenait plus. «Le Bon Dieu t'a donné un corps, il ne t'appartient plus. Tu vas devoir suivre les devoirs (sic) de ton mari», (paroles du curé citées par une répondante de Chicoutimi âgée de 97 ans). 
sent à croire que l'acquisition des connaissances se fondait sur l'expérience personnelle puisque, jusqu'à ce qu'elle donne naissance à son premier enfant, cette dame croyait que les bébés naissaient par le nombril. À cette époque, les tests de grossesse étaient inexistants. Une femme devinait alors son état par les nausées et/ou par l'arrêt de son cycle menstruel. D'autres témoignages confirment que tout ce qui concerne la naissance d'un enfant se passait sous silence. Par exemple, la future mère s'interdisait souvent d'annoncer son état à d'autres que son époux et sa mère. Puisque le secret de la naissance devait se perpétuer, la grossesse était dissimulée aux enfants ainsi qu'aux autres membres de la famille. À cette fin, la future mère portait des vêtements amples de même qu'un tablier et un corset. Le même désir de cacher la naissance du bébé incitait quelques informatrices à confectionner les vêtements de ce dernier en l'absence des enfants, lorsqu'ils étaient à l'école ou durant leur sommeil ${ }^{13}$.

C'est durant la grossesse que débutait la confection des trousseaux de semaine et de baptême. Le premier comportait l'ensemble des pièces vestimentaires d'usage courant, tels les bandes, corsets, couches, camisoles, jaquettes, robes, etc., et le second, les vêtements conçus spécialement pour la cérémonie baptismale. Au sujet de la confection, aucune règle ne précisait à quel moment elle devait commencer ni à quel moment elle devait être terminée.

Pour le trousseau de semaine, aucune norme ne déterminait le choix des tissus, la décoration et les modèles de ces vêtements. Il ressort cependant que l'ensemble des informatrices les confectionnaient dans des tissus blancs d'usage courant. Seulement quelques témoins confectionnaient des articles vestimentaires spécifiques pour le dimanche. Enfin, très peu d'entre elles avaient en leur possession des vêtements de couleur (rose ou bleu) dans le but de distinguer les filles des garçons. En fait, la ligne de conduite de l'époque incitait les mères à vêtir les bébés de robes blanches jusqu'à ce qu'ils soient en âge de marcher. C'est à ce moment-là seulement que les garçons commençaient à porter des culottes pour être différenciés des filles. Deux coutumes semblaient régir la transmission du trousseau de semaine. Premièrement, au sein de la famille immédiate où l'on privilégiait la fille aînée ou la première fille à se marier. Deuxièmement, lorsque cette norme n'était pas reconduite, le trousseau était destiné à une autre parente ou à une étrangère.

13. Ce trait a également été rapporté par Anne-Marie Desdouits pour certaines régions du Québec, op. cit., 27. 
Quant au trousseau de baptême, aucun modèle particulier n'était imposé pour sa confection. Par contre, toutes les répondantes s'entendaient sur un point: le trousseau devait être blanc. La pièce principale, la robe, arrivait aux chevilles de l'enfant ou lui dépassait les pieds. Le jupon, le manteau, la mante, le châle ainsi que le bonnet ou le châle de tête complétaient le trousseau. Toujours confectionné dans des tissus de qualité comme la soie, le coton glacé ou le satin, ce trousseau était réalisé avec beaucoup de soin. Le coût inhérent à sa réalisation importait peu étant donné qu'il servait lors d'un événement important et était susceptible d'être porté à quelques reprises. Enfin, peut-on parler de coutume de transmission lorsqu'on constate qu'il pouvait être transmis à la fille aînée, à une autre parente et même à une étrangère?

Durant la grossesse, la future mère était soumise à de nombreux interdits et prescriptions afin que l'accouchement se déroule normalement et que l'enfant naisse sans handicap physique ou psychologique. Pour contrer les fausses couches par exemple, il était déconseillé de sursauter ${ }^{14}$, de porter des talons hauts, de forcer, d'utiliser des produits de nettoyage toxiques, etc. ${ }^{15}$ Aussi, la femme enceinte était avertie que la fréquentation du cinéma, la lecture des journaux et la manière de s'asseoir pouvaient entraîner la naissance d'enfants handicapés. Enfin, elle apprenait aussi que porter des colliers, se mettre un fil autour du cou ou étendre du linge en passant les bras pardessus la corde étaient dangereux pour l'enfant, lequel risquait de naître «cordonné ${ }^{16}$ ».

Sur le plan de l'alimentation, peu de recommandations étaient faites si ce n'est d'éviter les aliments riches en matières grasses ainsi que la nourriture acide. Par contre, la future mère qui manifestait certains goûts bizarres en matière de nourriture devait les satisfaire par

14. Cette recommandation avait également cours en France et ailleurs au Québec. Pour la France, voir Catherine Fouquet et Yvonne Knibielher, L'histoire des mères du Moyen Age à nos jours (Paris, Montalba, 1980), 46; Jacques Gélis, op. cit., 121. Pour le Québec, se reporter à Suzanne Canuel, La grossesse et les phénomènes qui l'accompagnent dans la région de TroisPistoles: étude lexicale et aperçus ethnographiques, mémoire de maîtrise (linguistique), Université Laval, 1986, 25.

15. Au Saguenay, lorsqu'une femme était menacée de faire une fausse couche, on lui appliquait le remède de la soucoupe. Cela nécessitait l'utilisation de plusieurs ingrédients (alcool, oignon, œuf, etc.) variant au gré de chaque témoignage recueilli à ce sujet. Ce remède consistait à déposer sur le ventre de la femme enceinte une soucoupe remplie des produits ci-haut mentionnés. On lui prêtait en outre la propriété de soulager les douleurs "post-partum» et les descentes de matrice.

16. Le même genre de recommandations était véhiculé à l'extérieur du Saguenay. Sur cette question, voir Jean-Claude Dupont et Jacques Mathieu, Héritage de la francophonie canadienne: traditions orales (Québec, Presses de l'Université Laval, 1986), 58; Anne-Marie Desdouits, op. cit., 20; Jacques Gélis, op. cit., 134-135. 
crainte de les transmettre à l'enfant qu'elle portait. Il faut toutefois souligner que très peu de répondantes se soumettaient à cette prescription. De même, elles ne semblaient aucunement influencées par les risques présumément associés aux accidents climatiques en cours de grossesse ou durant l'accouchement, ou encore, par l'influence des «quêteux» susceptibles de jeter des sorts ${ }^{17}$.

Certaines conduites symboliques visant à déterminer le sexe du fœtus ainsi que le nombre d'enfants à naître ont été relevées au Saguenay. La pratique la plus répandue consistait à examiner l'abdomen de la femme enceinte. Certaines répondantes ont expliqué qu'un ventre pointu annonçait la naissance d'un garçon, tandis qu'un ventre rond indiquait la naissance d'une fille ${ }^{18}$. D'autres témoignages rapportent que les garçons étaient portés plus bas que les filles tandis que le ventre d'une femme enceinte d'une fille était plus petit. Enfin, quelques informatrices possédaient leurs propres recettes pour connaître le sexe de l'enfant qu'elles portaient. Pour l'une d'elles, les nausées et les vomissements annonçaient la naissance d'une fille. Pour une autre, ce sont les maux de bouche qui lui indiquaient la naissance d'une fille. Selon une troisième répondante, on pouvait prédire la naissance de jumeaux par la présence d'une barre verticale de couleur jaune sur l'abdomen.

\section{B - L'accouchement}

Les renseignements obtenus auprès de nos témoins suggèrent que l'accouchement se déroulait sensiblement de la même façon partout au Saguenay. De façon générale, la parturition s'effectuait en milieu familial (dans la chambre de la femme en couches), en présence du médecin ou plus rarement, en celui de la sage-femme ${ }^{19}$. Lors d'un

17. Il s'agit d'une croyance selon laquelle le quêteux avait le pouvoir de jeter des sorts à ceux et celles qui refusaient de le recevoir, et en particulier aux femmes enceintes. Se référer à Anne-Marie Desdouits, «La naissance dans le Québec traditionnel», Cap-aux-Diamants, 32 (hiver 1993): 14.

18. Ces conduites n'étaient pas spécifiques au Saguenay. Voir Diane Simoneau, Les médecines populaires au Québec (Montréal, Éditions Univers, 1980), 113; Mireille Laget, Naissance. L'accouchement avant l'âge de la clinique (Paris, Seuil, 1982), 86; Jacques Gélis, op. cit., 166; Suzanne Canuel, op. cit., 86.

19. Pour éviter toute confusion, précisons que la sage-femme se distinguait de l'assistante du médecin par le fait qu'elle pouvait effectuer seule des accouchements. L'assistante, pour sa part, exécutait seulement les consignes du médecin. Soulignons également qu'à l'époque de nos informatrices, l'intervention du médecin dans la pratique de l'accouchement et sa présence dans les maisons des parturientes était un phénomène encore nouveau. Plus récent encore est le transfert systématique des accouchements de la maison vers les hôpitaux. À cet égard, seulement deux informatrices sur 19 ont accouché à un moment ou l'autre de leur vie à l'hôpital. Ce fut, pour l'une, dès la naissance de son premier enfant et pour l'autre, lors de ses quatre derniers accouchements. En ce qui concerne l'influence du corps médical au sein de l'univers de la 
accouchement à la maison, la présence de visiteurs n'était pas bienvenue. On s'empressait en outre d'éloigner les enfants sous prétexte que la mère était malade, que le corbeau ou le sauvage ${ }^{20}$ allait passer et qu'ils ne devaient pas s'y trouver. Ils étaient alors envoyés chez une parente, une voisine ou tout simplement à l'extérieur de la maison.

Peu de personnes se trouvaient à la maison au moment de l'accouchement. Il arrivait que les parents ainsi que les beaux-parents de l'accouchée y soient, mais ils n'intervenaient pas directement dans la parturition. Il semble que la présence de ces personnes était fortuite car aucune règle ne la prévoyait, par exemple lors d'une première naissance ou, encore, lorsque l'accouchement s'avérait dangereux pour la santé de la mère et du bébé. En réalité, leur présence ou leur absence était déterminée beaucoup plus par des facteurs accidentels, comme le lieu de résidence et la disponibilité de chacun, que par un modèle culturel. D'ailleurs, certaines informatrices justifiaient la présence des parents ou des beaux-parents par le fait qu'elles et leurs époux cohabitaient avec eux au moment de la naissance du bébé. D'autre part, les informations obtenues auprès de nos répondantes établissent que les hommes - à l'exception du médecin - étaient rarement impliqués dans l'accouchement. Par exemple, seulement la moitié des conjoints se trouvaient dans la chambre à ce moment. L'absence de certains s'explique par le travail qui les retenait à l'extérieur, tandis que l'absence des autres était motivée par le fait qu'ils devaient accompagner les enfants chez un parent, un voisin, etc. Là encore, aucune règle ne semblait obliger le conjoint à demeurer auprès de son épouse.

Tous les intervenants étaient tenus d'ondoyer un bébé en danger de mort. À ce sujet, certaines répondantes ont raconté que lorsqu'il fallait baptiser un bébé sous condition, les hommes présents à l'accouchement avaient préséance sur les femmes. En cela, la population saguenayenne semblait se conformer à un comportement généralement admis au sein de la société québécoise: "Comme l'homme doit être préféré à la femme, le privilège de l'ondoiement lui

naissance, voir Jacques Gélis, op. cit., 16; Hélène Laforce, op. cit., 79 et autres; Andrée Lévesque, op. cit., 35-37. Sur la question de l'accouchement à l'hôpital, soulignons que ce n'est qu'en 1939 que l'Hôtel-Dieu de Chicoutimi ouvrit son département d'obstétrique. Sur ce sujet, se reporter à Normand Perron, Un siècle de vie hospitalière au Québec: les Augustines et l'Hôtel-Dieu de Chicoutimi 1884-1984 (Sillery, Presses de l'Université du Québec/Les Augustines de la Miséricorde de Jésus, 1984), 108.

20. On trouvera des explications similaires dans Suzanne Canuel, op. cit., 44; JeanClaude Dupont et Jacques Mathieu, op. cit., 59; Denise Lemieux et Lucie Mercier, «Familles et destins féminins. Le prisme de la mémoire, 1880-1940», Recherches sociographiques, 68,2-3 (1987): 267; Anne-Marie Desdouits, op. cit., 28. 
revient donc symboliquement, découlant de cette supériorité coutumière que lui reconnaît rituellement l'Église $(. . .)^{21} . »$

Dans un autre ordre d'idées, nous avons remarqué que le cycle lunaire suscitait des commentaires se rapportant au moment et au déroulement de l'accouchement. À titre d'exemple, on disait que la femme enceinte «allait à la lune», que «ça marchait à la lune», pour signifier qu'elle accoucherait lors d'une nuit de pleine lune ${ }^{22}$. De même, certaines informatrices estimaient qu'une femme qui accouchait dans ce contexte était privilégiée par rapport à celle dont la parturition survenait à un autre moment. Selon l'une d'elles, «la pleine lune, ça pouvait aider à l'accouchement, ça donnait des forces». Toutefois, aucune répondante ne croyait que le jour et la date pouvaient avoir une telle influence.

De façon générale, la parturition se déroulait selon un modèle uniforme. L'accouchernent avait lieu à la maison, la parturiente était installée sur son lit et la fenêtre de la chambre était fermée pour éviter les courants d'air ${ }^{23}$. L'accouchement qui survenait le jour ne différait en rien de celui ayant lieu la nuit, si ce n'est que certaines personnes prenaient la précaution de fermer la porte à clef. Sur la question des positions adoptées par les parturientes, l'enquête a permis d'établir que l'accouchement en position assise ou accroupie n'était pas connu de nos informatrices. La plus répandue consistait à s'allonger sur un lit, soit en position normale, soit en travers, de manière à ce que les pieds de l'accouchée reposent sur une chaise. Toutefois, lorsque le travail ne progressait pas, la parturiente pouvait accoucher debout en s'appuyant, par exemple, contre le cadre d'une porte. Enfin, quelques témoins ont mentionné la possibilité d'accoucher sur un lit dit «de misère» ou sur une paillasse à même le plancher ${ }^{24}$.

21. Hélène Laforce, op cit., 56. Cependant, dans les décrets et articles publiés par les autorités ecclésiastiques, rien n'empêchait les femmes d'ondoyer un enfant. «(...) en cas de nécessité, non seulement le prêtre ou le diacre, mais aussi un laïque, même une femme (...) peuvent baptiser (...)». On retrouve ce passage d'un décret du quatrième concile du Latran (1215) dans Louis Ott, Précis de théologie dogmatique (Mulhouse, Salvator, 1954), 499.

22. Des études récentes ont prouvé que cette croyance n'était pas fondée. À ce propos, voir Mireille Laget, op. cit., 124-125; Christian Hausser, Richard Bornais, Sylvie Bornais, «L'influence du cycle lunaire sur les accouchements», L'Union médicale du Canada, 114,7 (juillet 1985): 559; Raymond Roy, IREP (données non publiées).

23. La pratique consistant à fermer la fenêtre est peut-être l'héritage d'une croyance tombée en désuétude. Dans certaines régions de la France, cette conduite avait pour but de protéger le nouveau-né des sorciers, des magiciennes, etc. Sur cette question, voir Jacques Gélis, op. cit., 170; Mireille Laget, op. cit., 146-147.

24. L'expression pour désigner le lit de l'accouchement n'est pas unique au Saguenay. Mireille Laget, op. cit., 153, et Jacques Gélis op. cit., 263, en ont mentionné la présence dans certaines régions françaises. 
Aussitôt que les délivres étaient expulsés du corps de la mère, une des personnes présentes à l'accouchement les faisait brûler, les enterrait ou les jetait. Aucune des raisons invoquées par les témoins pour expliquer pourquoi et comment le placenta était détruit ne réfère à une symbolique quelconque. Seule la crainte qu'un enfant ou un animal trouvent les délivres ou que des odeurs désagréables se répandent motivait leur destruction, celle-ci devant avoir lieu dans les plus brefs délais. En outre, la plupart des informatrices n'accordaient aucune importance ou signification particulière aux délivres. Une répondante a cependant indiqué que l'on conservait un morceau de placenta pour frotter les taches de naissance afin de les faire disparaître. De même, l'eczéma dont souffrait le nouveau-né était également éliminé à l'aide d'un fragment de délivres.

En plus d'avoir recours aux services d'un prêtre lors de dystocies (accouchements difficiles), les informatrices ont laissé entendre que la femme en couches ainsi que certaines autres personnes priaient la bonne sainte Anne et sainte Marguerite Marie. En outre, l'utilisation d'objets à caractère religieux tels les calendriers et les images pieuses avait également cours dans les mêmes circonstances. Par contre, l'existence de dictons ou d'incantations à caractère magique ou autre n'a pas été attestée au Saguenay.

L'accouchement se soldant par la mort du fœetus était perçu comme un événement malheureux qui ne mettait cependant pas la responsabilité de la mère directement en cause. La morti-natalité était plutôt considérée comme un caprice de la nature ou un effet de la volonté divine. De plus, cet événement suscitait peu de commentaires étant donné que l'on devait taire autant un accouchement se terminant par le décès du bébé que la grossesse elle-même. Dans les cas où c'était la mère qui décédait, il était difficile de blâmer la sage-femme ou le médecin qui n'avait pu se rendre à temps auprès d'elle. En outre, les répondantes semblaient accepter la possibilité de mourir en accouchant comme un risque inhérent à la parturition. D'ailleurs, plusieurs d'entre elles connaissaient ou avaient entendu parler d'une femme décédée dans de telles circonstances ${ }^{25}$.

25. Cette réalité est rappelée par Danielle Gauvreau dans un article sur la mortalité maternelle. Voir «Donner la vie et en mourir: la mortalité des femmes en couches au Québec avant 1960», Dennis D. Cordell, Danielle Gauvreau, Raymond R. Gervais et Céline Le Bourdais, dirs., Population, reproduction, sociétés. Perspectives et enjeux de démographie sociale (Montréal, Presses de l'Université de Montréal, 1993), 235-256. 


\section{C - Le nouveau-né}

Dès sa naissance, l'enfant était souvent pris en charge par une proche de l'accouchée qui lui faisait sa toilette. Les personnes susceptibles de le faire étaient généralement la grand-mère maternelle ou paternelle, la sœur, la tante ou la cousine de la parturiente. Toutefois, ce pouvait tout aussi bien être la sage-femme, l'assistante, la releveuse, une voisine ou une amie. Par conséquent, à part le fait que c'était toujours une femme qui lavait le bébé, il ne semblait pas y avoir de règle déterminant qui de la parenté ou de l'entourage devait accomplir cette tâche. De même, aucune modalité ne déterminait la présentation du bébé à la mère. Le moment de le présenter, la façon de le faire et la personne chargée de le faire n'étaient soumis à aucune règle particulière. Ainsi, c'était généralement la grand-mère du bébé qui le présentait à sa mère. Il pouvait aussi arriver que le médecin, la sage-femme ou l'assistante remplisse cette fonction. D'autre part, le rituel saguenayen ne prévoyait pas un ordre de présentation du bébé allant, par exemple, de la mère aux autres personnes présentes dans la maison. De même, aucun geste à caractère symbolique n'était posé par la mère lorsqu'elle voyait son bébé pour la première fois. Une informatrice a toutefois mentionné qu'elle s'interdisait de l'embrasser avant son baptême, prétextant qu'il ne lui «appartenait pas encore». Les codes culturels saguenayens n'étaient guère plus élaborés lorsqu'arrivait le moment d'annoncer la naissance de l'enfant. C'était l'époux ou un autre parent de l'accouchée, voire le médecin ou la voisine qui se chargeait de la nouvelle. Pour cette annonce, aucune formulation stéréotypée, démontrant par exemple une préférence pour les garçons ou pour les filles, n'était attestée au Saguenay.

$\mathrm{Au}$ moment de la toilette du nouveau-né, il arrivait qu'on lui coupe le filet de la langue pour éviter qu'il bégaie ${ }^{26}$. De même, nous avons relevé certains comportements symboliques pour la coupe des ongles et des cheveux du bébé. Ainsi, la crainte que les ongles épaississent, que l'enfant perde des forces ou qu'il soit moins intelligent incitait quelques témoins à observer les prescriptions véhiculées en ce sens. Enfin, parce qu'elle craignait que ses enfants soient moins intelligents, une informatrice ne leur coupait pas les cheveux. Cependant, aucune répondante n'a mentionné jusqu'à quel âge elles devaient respecter ces prescriptions.

À l'exception des commentaires formulés à propos de la ressemblance du bébé avec ses parents, aucun présage relié, par exemple, aux

26. C'est aussi ce qu'on observait dans certaines régions de la France (Françoise Loux, Le jeune enfant et son corps dans la médecine traditionnelle (Paris, Flammarion, 1978), 128. 
doigts du bébé ou à une autre partie de son corps n'a été mentionné par les informatrices. Ces dernières accordaient toutefois de l'importance aux taches de naissance, lesquelles semblaient consécutives aux peurs et/ou aux envies alimentaires ressenties par une femme durant sa grossesse ${ }^{27}$. Par exemple, la fille d'une informatrice avait une tache grise sur une cuisse parce qu'au cours de sa grossesse, elle avait touché à cette partie de son corps à la vue d'une souris.

Pour soigner un enfant malade, plusieurs mères recouraient à la Vierge, à sainte Anne et à saint Joseph. L'utilisation des médailles et le fait de vouer les enfants à un saint ${ }^{28}$ étaient également pratique courante au Saguenay. Soulignons enfin que le recours à une guérisseuse a été attesté par certaines de nos informatrices. L'histoire d'un enfant guéri de sa jaunisse après que sa mère eut envoyé une couche imbibée d'urine à la guérisseuse confirme l'existence de tels recours. En ce qui concerne les problèmes causés par la diarrhée, aucun soin à caractère religieux ou symbolique ne semblait prévu. Pour soigner cette affection, la mère avait le choix entre les trois recours suivants: $1^{\circ}$ interrompre l'allaitement et nourrir l'enfant au lait de vache; $2^{\circ}$ faire bouillir le lait de vache et y mettre des gouttes prescrites par le médecin; $3^{\circ}$ interrompre toute forme d'alimentation lactée et faire boire à l'enfant de l'eau de riz ou des tisanes.

\section{D - Les relevailles et l'allaitement}

La naissance d'un enfant donnait lieu à une période de repos et de récupération appelée les relevailles ou les «40 jours». Cette période comportait deux phases, l'alitement d'une durée de neuf jours et la récupération. Au cours de la première phase, la mère devait rester alitée, faire des siestes et éviter la lecture, car c'était dangereux pour la vue. Pour expliquer aux enfants les raisons de l'alitement, on invoquait la maladie de la mère, «la jambe cassée», etc.

$\mathrm{Au}$ terme de ces neuf jours de repos, la mère reprenait progressivement ses diverses occupations. Cette seconde phase, dite de récupération, comportait aussi des interdits et des prescriptions auxquels se soumettait la mère afin d'éviter certaines complications, telle la reprise des pertes sanguines, les maux de ventre et autres. Pour ce faire, il ne fallait pas laver les planchers ni laver dans «l'eau forte», pas plus que forcer ou étendre du linge. En outre, il était recommandé

27. Cette relation de cause à effet est également mentionnée par Pierre Desruisseaux, op. cit., 121; Sœur Marie Ursule, «Civilisation traditionnelle des Lavalois», Les Archives de folklore (Québec, Presses de l'Université du Québec), 5,6 (1951): 93; Françoise Loux, op. cit., 56-60.

28. On ne faisait porter à l'enfant que des vêtements aux couleurs du saint. 
de se bander le ventre ou de porter un corset et d'éviter les refroidissements.

Pour qu'elle puisse compléter ses «40 jours», la mère recevait l'aide d'un tiers. Ce rôle revenait souvent à sa mère, à sa belle-mère ou à une de ses sœurs. Lorsque ces personnes n'étaient pas disponibles, le rôle de releveuse était imparti à une autre parente, à une voisine ou à celle qui avait assisté l'accoucheur. Lorsque le réseau d'entraide faisait défaut, certaines femmes se voyaient contraintes d'engager une servante pour la totalité ou une partie des relevailles.

Lorsqu'une femme «relevait», son époux ne pouvait pas l'obliger à avoir des relations sexuelles. Quelques informatrices ont expliqué qu'elles avaient le droit de se refuser à leur mari durant ces «40 jours», cela étant même approuvé par l'Église. En dehors de cette période, celles qui ne faisaient pas «leur devoir conjugal» devaient s'en confesser. Une informatrice a raconté qu'un prêtre l'avait excommuniée pour cette raison ${ }^{29}$. À la fin des « 40 jours», l'homme et la femme devaient «laisser faire la nature» et le «Bon Dieu». Mis à part l'abstinence et l'allaitement prolongé, les moyens de contraception comme le coït interrompu ou la méthode Ogino étaient interdits par l'Église. À cet égard, il semble que les visites du prêtre à la maison, la menace de refus des sacrements ainsi que les sermons lors des retraites paroissiales constituaient des moyens efficaces pour inciter les couples à avoir d'autres enfants.

Les relevailles complétées, plusieurs femmes se rendaient à la messe pour remercier le «Bon Dieu d'être encore vivante». Ce comportement semble être le reliquat d'un rite de passage très ancien. En France, par exemple, la fin de cette période était soulignée à l'église lors de la cérémonie des relevailles. Cette célébration très élaborée permettait à la femme de réintégrer la société dont elle était exclue au cours de la grossesse ${ }^{30}$.

Dans un autre ordre d'idées, l'enquête sur le terrain a permis d'établir que nos informatrices étaient les dernières représentantes d'une époque où l'allaitement au sein était privilégié par rapport au biberon. Près de la moitié des témoins considéraient l'allaitement comme un devoir, comme quelque chose de naturel qui était préférable pour le bébé. Pourtant, après avoir allaité un, deux ou plusieurs

29. Il est probable que cette femme s'était plutôt vue refuser les sacrements.

30. Voir Arnold Van Gennep, Manuel de folklore français contemporain, I,1: Du berceau à la tombe (Paris, Éditions A. et J. Picard et Cie, 1943), 119; Jacques Gélis, op. cit., 292-294; Antoinette Fauve-Chamoux, Cultural Influences on Demographic Change, texte d'une communication présentée au colloque de la «Social Science History Association», Washington, DC (novembre 1989): 16-19; Anne-Marie Desdouits, Le monde de l'enfance..., 29. 
bébés, un certain nombre avaient finalement opté pour le biberon, et cela même si elles étaient conscientes que l'allaitement maternel permettait d'espacer les naissances. Les raisons invoquées par ces personnes afin d'expliquer leur préférence pour le biberon faisaient état de plaies aux seins, de problèmes causés par les engorgements, d'une surcharge de travail, etc.

Selon les témoins qui ont allaité quelques-uns de leurs enfants, le nombre de mois consacrés à l'allaitement variait selon les naissances. Dans l'ensemble, la durée de l'allaitement variait de deux mois à deux ans et était laissée à la discrétion de la mère. Pour mettre un terme à l'allaitement, il était recommandé d'utiliser la purgation ou des bandes enserrant les seins. Il existait aussi un recours consistant à placer un bouchon de liège entre les seins. Celles qui interrompaient l'allaitement devaient le faire de manière progressive, car un arrêt trop brusque risquait d'entraîner une phlébite (caractérisée par les expressions avoir des «jambes de lait» ou dont le lait était «tombé dans les jambes»). Au Saguenay, il y avait au moins deux façons de soigner les femmes aux prises avec ce genre de problème. En premier lieu, on faisait ingurgiter à la mère un remède à base de vin de messe et de lait chaud. On utilisait aussi des feuilles de tabac préalablement trempées dans du vinaigre chaud que l'on appliquait sur les jambes de la malade.

\section{E - Le baptême}

Dès la naissance de l'enfant, il fallait s'empresser de le faire baptiser car on craignait qu'il meure avant d'avoir reçu les premiers sacrements. Un enfant mort sans baptême affligeait les parents parce qu'ils croyaient que le bébé séjournait dans les limbes, cet endroit où les enfants étaient privés de la vue du «Bon Dieu». C'est probablement pour cette raison qu'on baptisait presque tous les enfants dans les 48 heures suivant leur naissance (G. Bouchard, données non publiées) $)^{31}$.

Par ailleurs, le baptême d'un enfant ne donnait pas lieu au déploiement d'un rituel sophistiqué. Au Saguenay, en effet, le «compérage» se déroulait selon un canevas très simple. De manière générale, le parrain et la marraine se rendaient chez les parents de leur filleul(e) avant le baptême. De là, il n'y avait pas formation de cortège pour se

31. La menace des limbes constituait probablement un excellent moyen de pression utilisé par les prêtres, afin que les parents ne dépassent pas le délai de trois à huit jours prescrit par les autorités ecclésiastiques. Voir l'article 452 de l'Acta et decreta Concilii plenarii quebecensis primi, Anno Domini, MCMIX, Québec, L'Action Sociale (1912): 711 p. 
rendre jusqu'à l'église. Tout au plus, deux véhicules non décorés voiture(s) à cheval ou automobile(s) - dans lesquels prenaient place le père, l'enfant, le parrain, la marraine et la porteuse. Aucun arrêt ne marquait le trajet jusqu'à l'église. Par contre, le retour comportait parfois une halte au magasin général, laquelle semblait dictée par l'obligation qu'avait le parrain d'acheter un cadeau à la mère, au bébé, à la marraine ou à la porteuse. Il arrivait aussi que le parrain défrayait les coûts de la fête suivant le baptême. Mais le principal engagement du parrain consistait à faire sonner les cloches à la fin de la cérémonie. Selon plusieurs informatrices, cette dernière obligation était très importante, car en l'absence de la sonnerie des cloches, l'enfant risquait de devenir sourd ${ }^{32}$.

La cérémonie du baptême avait lieu dans la plus stricte intimité, en présence du père, des parrain et marraine et de la porteuse. Les grands-parents, les oncles et les tantes, les frères et les sœurs du bébé ainsi que les voisin(e)s et les ami(e)s y assistaient rarement. À une exception près, le déroulement de la cérémonie religieuse n'était le lieu d'aucun présage se rapportant aux faits et gestes de l'enfant. Selon une répondante, lorsque le prêtre versait l'eau sur la tête de l'enfant, ce dernier avait le réflexe de bâiller; c'était l'instant choisi par le diable pour sortir de son corps.

Une réception donnée au domicile des parents suivait la cérémonie baptismale. Il s'agissait généralement d'un souper et d'une veillée de cartes. Le repas réunissait de cinq à vingt personnes, soit le parrain, la marraine, la porteuse, les frères et les sœurs de l'enfant, les grands-parents ainsi que, exceptionnellement, les oncles et les tantes. Tout ce qui se rapportait à cette réception, à savoir le nombre d'invités, l'identité des personnes invitées et la formulation des invitations (par écrit ou de vive voix) était laissé à la discrétion des parents.

Le baptême était le prétexte au resserrement des liens familiaux puisque le choix du parrain, de la marraine et de la porteuse s'effectuait surtout au sein de la parentés3. D'autre part, le choix du parrain

32. Une croyance similaire a également été rapportée par Françoise Loux, Le jeune enfant..., 146, pour certaines régions de la France et par Anne-Marie Desdouits, op. cit., 48, pour d'autres régions du Québec.

33. Entre 1920 et 1950 au Saguenay, les parrains et marraines étaient choisis au sein de la parenté dans une proportion de $68,2 \%$ et $74,3 \%$, respectivement. En outre, les premiers résultats d'une étude portant sur les modèles de parrainage confirment la tendance à privilégier des membres de la famille proche. En effet, entre 1882 et 1895 à Notre-Dame de Laterrière (Saguenay), 75\% des parrains et marraines étaient apparentés à leurs filleul(e)s. Entre 1942 et 1955, cette tendance s'est généralisée, devenant ainsi la norme en usage dans la société saguenayenne, alors que $99 \%$ des parrains et marraines étaient des parents de l'enfant (recherche en cours à l'IREP). 
et de la marraine pouvait donner lieu à un rite matrimonial consistant à «moucher le filleul». Non seulement on choisissait alors un parrain et une marraine étrangers l'un à l'autre, mais, à la fin de la cérémonie, le parrain était invité à embrasser la marraine.

Au Saguenay, on disait qu'une personne «lâchait la queue de la chatte» lorsqu'elle était dans «les honneurs» pour la première fois. Selon une informatrice, le jeune homme célibataire qui «lâchait la queue de la chatte» avait désormais le «droit d'aller voir les filles et de se marier». Il arrivait qu'on souligne cet événement après la cérémonie du baptême en faisant sortir un chat noir de la maison à l'arrivée du parrain et de la marraine. D'autre part, l'expression consacrée pour désigner l'homme ou la femme qui était dans «les honneurs» uniquement en vertu d'un lien matrimonial était «parrain salop», «marraine salope». À ce sujet, certains témoins circonstanciels ont mentionné l'existence des expressions «parrain cochon» et «marraine cochonne», lesquelles faisaient également référence à ce type de parrainage.

Le jour du baptême, on donnait à l'enfant entre deux et cinq prénoms choisis par ses parents ou par ses parrain et marraine. Le choix des prénoms s'effectuait suivant certains modèles. En premier lieu, il était possible de prendre le prénom du saint ou de la sainte dont le jour anniversaire correspondait à la date de naissance de l'enfant. En deuxième lieu, les prénoms de ce dernier pouvaient être choisis dans le but de remercier ou d'honorer quelqu'un, par exemple un membre de la famille ${ }^{34}$, un prêtre, le médecin qui avait procédé à l'accouchement et ainsi de suite. En dernier lieu, le choix des prénoms pouvait varier en fonction des goûts de la mère ou de toute autre personne habilitée à effectuer ce choix.

\section{III - COMPARAISON SAGUENAY/CHARLEVOIX}

L'un des objectifs de cette enquête consistait à comparer les rituels entourant la naissance au Saguenay et dans Charlevoix. Rappelons que le but de cette comparaison était d'établir si les mouvements migratoires depuis Charlevoix au cours du XIX Xiècle $^{\mathrm{e}}$ avaient amené des changements dans les contenus coutumiers au Saguenay. Les changements ainsi engendrés pouvaient prendre deux trajectoires différentes, à savoir la «déritualisation» ou la contraction

34. Il a été établi que $48 \%(\mathrm{~N}=72)$ des enfants de nos informatrices portaient le prénom d'un membre de la famille proche. Parmi les garçons, $41,7 \%(\mathrm{~N}=30)$ étaient prénommés comme leur parrain, $9,7 \%(\mathrm{~N}=6)$ comme leur père et $6,9 \%(\mathrm{~N}=5)$ comme un de leurs grands-pères. Dans le cas des filles, 33,3\% ( $\mathrm{N}=24)$ avaient reçu le prénom de leur marraine, $8,3 \%(\mathrm{~N}=6)$ celui d'une de leurs grands-mères et $1,3 \%(\mathrm{~N}=1)$ celui de leur mère. 
et le renouvellement, ce dernier étant susceptible de modifier le rituel de deux manières, soit par expansion (inflation et ajout), soit par réaménagement (redéfinition, substitution). Ces deux types de changements ont effectivement été relevés au Saguenay, mais les modifications observées n'affectent que certains éléments du rituel. Comme nous allons le constater, l'approche comparée a surtout permis de démontrer que c'est tout le noyau du rituel original qui a été réimplanté au Saguenay, assorti de quelques variantes.

\section{A - «Déritualisation»}

Le concept de «déritualisation» suggère que des traits coutumiers constatés dans Charlevoix étaient absents au Saguenay. Deux formes d'érosion pouvaient affecter les codes culturels initiaux. Il s'agit d'abord de la disparition complète d'une norme, d'une croyance ou d'un rite, et ensuite de l'absence d'au moins un des éléments du rite (dans ce cas, on parlera d'érosion partielle).

L'absence au Saguenay de certains rites et croyances a été observée sur trois points. Contrairement aux répondantes de Charlevoix, celles du Saguenay ne croyaient pas qu'il puisse être dangereux d'accoucher un vendredi 13. De même, il n'était pas dans l'ordre des choses de porter le deuil à la suite d'une fausse couche ni d'essayer de transférer la fécondité de la nouvelle mère aux femmes stériles ou nouvellement mariées, en leur faisant porter le nouveau-né. Ce genre de rites, relevé dans les années 1940 aux Éboulements, se caractérisait par l'expression "passer le château ${ }^{35}$ ».

En outre, la disparition de certains éléments d'un rite a été constatée à quelques reprises, notamment dans les coutumes et les croyances entourant le parrainage, dans les présages formulés au moment du baptême ainsi que dans les croyances associées à la sonnerie des cloches à la fin de la cérémonie du baptême.

\section{$B$ - Renouvellement par expansion et/ou réaménagement}

Le renouvellement par expansion et/ou réaménagement est le deuxième type de changement ayant pu modifier le rituel charlevoisien lors de son implantation au Saguenay. C'est peut-être parce qu'il implique à la fois l'expansion et la substitution que ce processus a apporté le plus de bouleversements au sein du rituel relié à la naissance. Mise à part l'évolution observée par le biais de l'expansion,

35. À ce sujet, voir Antoine Tremblay, Sur le plateau laurentien. Analyse sociale d'une communauté rurale. Notre-Dame-de-l'Assomption des Éboulements, mémoire de maîtrise (sciences sociales), Université Laval, 1948, 70. 
nous avons constaté que le processus de renouvellement par réaménagement avait pris deux formes. La première se rapporte aux traits coutumiers renouvelés à la suite du remplacement de l'ensemble de leurs variantes. La seconde concerne les pratiques rituelles dont au moins une des variables a été remplacée par une autre.

\section{1 - Expansion par intensification et ajout}

Peu d'indices laissent croire à des modifications allant dans le sens de l'intensification. Au Saguenay, nous recherchions les signes de la prédominance des conduites associées à la religion. L'influence de cette dernière et le respect des informatrices pour tout ce qui la concerne ressortent clairement lors des entrevues. Dans la sexualité, par exemple, où les tabous et le contrôle exercé par l'Église étaient omniprésents; ensuite dans les comportements de la femme enceinte qui se recommandait à la Vierge et à la bonne sainte Anne pour que l'enfant naisse sans aucun handicap. L'omniprésence de la religion transparaît également au moment de la parturition alors que l'on recourait à des objets sacrés, tel le crucifix ou le calendrier afin que tout se déroule sans problème. Toutefois, en raison de l'inégalité des questionnaires utilisés et du nombre d'enquêtes effectuées de part et d'autre, il n'a pas été possible de vérifier s'il en était de même dans Charlevoix.

La présence de rites et de croyances imputables au processus d'expansion par innovation et/ou emprunt a été vérifiée à certaines reprises. D'abord, les expressions «parrain cochon» et «marraine cochonne» pour désigner le parrainage indirect semblaient connues seulement des résidentes du Saguenay. De même, le rite incitant le parrain à embrasser la marraine (que les Saguenayens caractérisaient par l'expression «moucher le filleul») n'a pas été retrouvé dans Charlevoix. D'autre part, les informatrices des deux régions s'entendaient pour dire que «c'est toujours pendant les tempêtes que les femmes accouchent». Mais dans Charlevoix, on ne connaissait pas l'expression «ça va être la tempête à une telle», laquelle a été mentionnée à quelques reprises au Saguenay. De même, les commentaires formulés pour expliquer aux enfants l'alitement de leur mère ont fait l'objet d'une certaine expansion. Dans les deux aires d'observation, on invoquait la maladie de la mère et «la jambe cassée». Mais au Saguenay, on expliquait également aux enfants que c'était le médecin qui avait cassé la jambe de leur mère. Ceci ne prouve pas pour autant qu'il y ait eu expansion du rituel au Saguenay; il s'agit de cas isolés, et il est possible que les informatrices de Charlevoix aient tout simplement omis de donner certains renseignements. 


\section{2 - Réaménagement par remplacement ou substitution totale}

La première forme de renouvellement par réaménagement se rapporte aux contenus coutumiers dont tous les éléments originaux ont été remplacés par des variantes saguenayennes. Pour illustrer ce phénomène, reprenons trois des six cas relevés lors de l'analyse. Ainsi, au Saguenay, on utilisait l'expression «les 40 jours» pour désigner les relevailles, alors que dans la région de Charlevoix, c'est par les termes «relevage» et «quarantaine» qu'on référait à cette période.

Aussi, la visite des parents, des voisins et des amis après la naissance leur permettait de commenter de multiples façons l'apparence du nouveau-né. Au Saguenay, les visiteurs formulaient seulement des commentaires se rapportant à la ressemblance du bébé avec ses parents. Une informatrice saguenayenne a toutefois mentionné qu'un enfant qui naissait avec beaucoup de cheveux serait quelqu'un de très fort. D'un autre côté, aucun des présages relevés par Gagnon dans Charlevoix ne semblait véhiculé au Saguenay. Par exemple, aucune des répondantes saguenayennes n'a mentionné que les visiteurs commentaient la longueur et la forme des doigts du bébé, la forme de la langue, etc. ${ }^{36}$

Enfin, au Saguenay, le choix du parrain et de la marraine s'effectuait selon deux modèles différents. D'abord, on privilégiait des personnes apparentées à l'enfant. Ensuite, les couples mariés avaient préséance sur les célibataires. Dans la région de Charlevoix, deux autres modèles déterminaient ce choix. Le premier incitait les parents à ne désigner que le parrain, laissant à ce dernier le choix de la marraine. En outre, les parents ne devaient pas opter pour des couples mariés ou sur le point de l'être afin que leur enfant «ait deux parrains et deux marraines ${ }^{37}$ ».

\section{3 - Réaménagement par substitution partielle}

La seconde forme de renouvellement implique à la fois les processus d'érosion partielle et de substitution. Ce type particulier s'observe parmi les conduites rituelles qui, dans Charlevoix, étaient déjà très ramifiées. Au Saguenay et dans Charlevoix, toutes les futures mères étaient soumises à des interdits et des prescriptions afin d'éviter les fausses couches ainsi que la naissance d'un enfant handicapé ou «cordonné». Lors de l'enquête saguenayenne, nous avons relevé

36. Dans Charlevoix, un enfant aux doigts longs «(serait) grand, (jouerait) de la musique ou (ferait) de grandes choses. De même, celui dont les doigts étaient «croches» ou «retroussés» serait musicien et ainsi de suite. Voir Jean-Philippe Gagnon, Rites et croyances..., 113.

37. Ibid., 105. 
17 recommandations à ce propos. De ce nombre, dix étaient communes aux deux aires d'observation, tandis que les sept autres étaient spécifiques au Saguenay ${ }^{38}$. Ainsi, on conseillait aux futures mères de cette région d'éviter de porter des talons hauts, de s'étirer, de forcer, de laver des murs, des plafonds ou des planchers, de lire les journaux, d'aller au cinéma et de passer les bras par-dessus la corde à linge. Ces éléments vont dans le sens d'une expansion du rituel. Toutefois, sur d'autres points, le rituel est simultanément affecté par le processus de contraction. En effet, l'existence d'une mise en garde recommandant aux femmes enceintes d'éviter de regarder une «créature morte» si elles ne voulaient pas accoucher d'un mort-né semblait inconnue au Saguenay. Les témoins du Saguenay ignoraient également que l'image de l'Enfant Jésus et les photos de famille avaient un effet bénéfique sur la formation du fœtus.

De même, pour éviter que les jeunes enfants sachent la vérité sur la grossesse et la naissance, les rituels du Saguenay et de Charlevoix comportaient diverses explications. Dans ces deux régions, le passage du corbeau et le cadeau fait par le Petit Jésus expliquaient la présence du bébé dans la maison. On retrouve aussi au Saguenay des explications inconnues dans Charlevoix, soit l'intervention du «Bon Dieu», et le passage de la cigogne, du «sauvage», du «corbeau qui va vous dévorer ou vous crever les yeux» et de la «mi-carême». En contrepartie, aucune informatrice saguenayenne n'a mentionné que «c'est la femme qui a apporté le bébé ${ }^{39}$ ». Cet exemple et celui qui précède illustrent bien de quelle façon le phénomène de substitution s'est doublé d'une diversification à l'échelle des variantes.

\section{CONCLUSION}

L'objet de cet article visait à connaître certains aspects de la ritualité véhiculée au Saguenay et dans Charlevoix au cours de la première moitié du $\mathrm{XX}^{\mathrm{e}}$ siècle. Pour ce faire, nous avons décrit le rituel saguenayen de la naissance, lequel a par la suite été comparé à celui de Charlevoix afin d'identifier des points de ressemblance et de dissemblance. Rappelons que cette partie s'appuyait sur la problématique culturelle des transferts migratoires et de leurs conséquences sur les

38. Dans les deux régions, il était recommandé à la femme enceinte d'éviter les chutes, de lever des objets lourds, d'utiliser des produits nettoyants toxiques, de voyager, de voir un handicapé, de s'asseoir une jambe repliée sous elle, d'avoir un fil autour du cou, de porter un collier et, enfin, de sursauter puis de se toucher à la vue d'un objet, d'un animal ou d'une personne. De même, il lui était conseillé de regarder des images pieuses afin que son enfant naisse sans handicap.

39. Jean-Philippe Gagnon, op. cit., 100. 
contenus coutumiers. Enfin, les résultats de cette étude allaient permettre d'en connaître un peu plus sur la question de la différenciation des aires culturelles.

Mentionnons d'abord que la comparaison effectuée entre les deux corpus de données a permis d'établir que la plupart des composantes attestées au Saguenay le sont également dans Charlevoix. Ce résultat va à l'encontre de notre hypothèse de départ, laquelle reposait sur les mutations du rituel de la naissance consécutives aux transferts de population. En effet, l'homogénéité des rituels a été vérifiée à plusieurs reprises, notamment dans les modalités associées à l'annonce de la grossesse, dans les normes entourant les façons de s'habiller pour la dissimuler, dans les règles déterminant le nombre de personnes présentes dans la chambre lors de la parturition, et ainsi de suite. Par ailleurs, seulement quelques traits coutumiers ne sont pas véhiculés au Saguenay, tandis que d'autres conduites symboliques attestées dans Charlevoix ont subi des modifications qui traduisent un certain renouvellement. Nous avons pu confirmer l'absence au Saguenay de certains rites (par exemple, la règle incitant les femmes à porter le deuil à la suite d'une fausse couche) ou d'éléments d'un rite, comme l'absence d'une des variantes entourant la sonnerie des cloches au baptême. D'un autre côté, très peu d'indices laissent supposer que le processus d'expansion a affecté le rituel initial, si ce n'est l'introduction possible du rite incitant le parrain à embrasser la marraine à la fin de la cérémonie du baptême («moucher le filleul»). En fait, c'est le processus de renouvellement qui semble avoir eu le plus d'impact sur le rituel initial, ce qui s'est traduit de plusieurs façons: dans les expressions référant à la période des relevailles, dans les interdits et recommandations formulés à la future mère pour éviter que l'enfant naisse avec des handicaps ou que l'accouchement se double de complications, dans les coutumes relatives au choix du parrain et de la marraine, etc.

En somme, ce qui semble ressortir assez clairement, c'est l'existence d'une structure commune aux deux régions et sur laquelle se greffent parfois des éléments de différenciation que l'on pourrait attribuer aux mutations consécutives aux mouvements de population. Il faut toutefois demeurer circonspect dans l'interprétation des données, car il est possible que ces variantes soient tout simplement le fruit d'omissions de la part des informatrices de l'une ou l'autre région. Bien que nos observations laissent croire que le Saguenay constitue le prolongement culturel de l'espace charlevoisien, une certaine prudence est toujours de mise. Des études complémentaires, à l'aide d'autres indicateurs, seraient utiles sinon nécessaires avant de conclure définitivement sur cette question. Toutefois, notre enquête 
permet d'enrichir la problématique de la stratification spatiale et de faire ressortir l'importance que prenait la naissance d'un enfant au Saguenay et dans Charlevoix. 